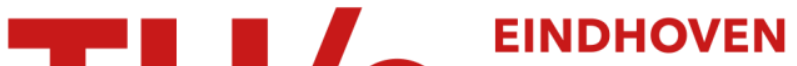 \\ UNIVERSITY OF \\ TECHNOLOGY
}

\section{Exploring cooperative fitness tracking to encourage physical activity among office workers}

\section{Citation for published version (APA):}

Ren, X., Yu, B., Lu, Y., \& Brombacher, A. C. (2018). Exploring cooperative fitness tracking to encourage physical activity among office workers. Proceedings of the ACM on Human-Computer Interaction, 2(CSCW), [146]. https://doi.org/10.1145/3274415

\section{Document license:}

CC BY-NC-ND

DOI:

$10.1145 / 3274415$

Document status and date:

Published: 01/11/2018

\section{Document Version:}

Accepted manuscript including changes made at the peer-review stage

\section{Please check the document version of this publication:}

- A submitted manuscript is the version of the article upon submission and before peer-review. There can be important differences between the submitted version and the official published version of record. People interested in the research are advised to contact the author for the final version of the publication, or visit the $\mathrm{DOI}$ to the publisher's website.

- The final author version and the galley proof are versions of the publication after peer review.

- The final published version features the final layout of the paper including the volume, issue and page numbers.

Link to publication

\section{General rights}

Copyright and moral rights for the publications made accessible in the public portal are retained by the authors and/or other copyright owners and it is a condition of accessing publications that users recognise and abide by the legal requirements associated with these rights.

- Users may download and print one copy of any publication from the public portal for the purpose of private study or research.

- You may not further distribute the material or use it for any profit-making activity or commercial gain

- You may freely distribute the URL identifying the publication in the public portal.

If the publication is distributed under the terms of Article 25fa of the Dutch Copyright Act, indicated by the "Taverne" license above, please follow below link for the End User Agreement:

www.tue.nl/taverne

Take down policy

If you believe that this document breaches copyright please contact us at:

openaccess@tue.nl

providing details and we will investigate your claim. 


\title{
Exploring Cooperative Fitness Tracking to Encourage Physical Activity among Office Workers
}

\author{
XIPEI REN, Eindhoven University of Technology \\ BIN YU, Eindhoven University of Technology \\ YUAN LU, Eindhoven University of Technology \\ AARNOUT BROMBACHER, Eindhoven University of Technology
}

\begin{abstract}
This paper presents a field study on using peer-based cooperative fitness tracking (PCFT) to promote workplace fitness. The social bonding achieved through a collective fitness goal and the sharing of fitness data between two co-workers has been explored as a motivational factor that can encourage physical activity. The study involved 10 dyads of co-workers in two groups (a distributed vs. a co-located group) based on their proximity at work. The effectiveness of the proposed PCFT was examined by comparing fitness data over a period of three weeks: the baseline week, the PCFT intervention week, and the postintervention week. The proximity effects on PCFT were investigated by comparing the fitness data, goal commitment, and interview results between the two groups. The quantitative results showed that the physical activity of participants in the co-located group improved significantly after the PCFT intervention. The qualitative results suggested that PCFT may improve the awareness of being physically active, stimulate exchange of knowledge to support active lifestyles and facilitate including fitness breaks in the daily work routine. Based on these findings, we discuss design implications for the future development of the PCFT-based applications and their potential contribution to increased office vitality.
\end{abstract}

CCS Concepts: $\bullet$ Human-centered computing $\rightarrow$ Collaborative and social computing $\rightarrow$ Empirical studies in collaborative and social computing

\section{KEYWORDS}

Cooperative fitness tracking; office worker; promotion of physical activity; proximity effects.

\section{ACM Reference format:}

Xipei Ren, Bin Yu, Yuan Lu, Aarnout Brombacher. 2018. Exploring Cooperative Fitness Tracking to Encourage Physical Activity among Office Workers. PACM on Human-Computer Interaction. Volume 2, CSCW. Article 146 (November 2018), 18 pages. https://doi.org/10.1145/3274415

\section{INTRODUCTION}

The rapid advance of office automation has substantially reduced physical demands while increasing the mental workload in many jobs. This transition has been closely associated with prolonged sedentary time and insufficient physical activity among workers, leading to higher risks of developing various chronic conditions, such as obesity, diabetes, and cardiovascular diseases [50]. Increasingly, fitness tracking technologies (e.g., activity trackers) have been introduced into the work environment and in some cases they are now seen as office necessities. It is reported that 10,000 companies in the US provided activity trackers to their employees in 2014 [61]. Moreover, it is expected that more than 13 millions of these devices will

\footnotetext{
Permission to make digital or hard copies of all or part of this work for personal or classroom use is granted without fee provided that copies are not made or distributed for profit or commercial advantage and that copies bear this notice and the full citation on the first page. Copyrights for components of this work owned by others than the author(s) must be honored. Abstracting with credit is permitted. To copy otherwise, or republish, to post on servers or to redistribute to lists, requires prior specific permission and/or a fee. Request permissions from Permissions@acm.org.

2573-0142/2018/November - $146 \$ 15.00$

Copyright is held by the owner/author(s). Publication rights licensed to ACM.

https://doi.org/10.1145/3274415
}

PACM on Human-Computer Interaction, Vol. 2, No. CSCW, Article 146. Publication date: November 2018. 
be incorporated into workplace wellness programs by the end of 2018 [39]. Tracking and providing feedback on daily activities is beneficial for the engagement of individuals in physical mobility and an active lifestyle but it seems to be challenging to get users to adhere to using fitness tracking devices [7]. To facilitate the use of fitness tracking devices in everyday work, corporations have started to try various motivational strategies, such as short-term challenges [22], financial incentives [40], and social competitions [21].

Social interactions can play a crucial role in the adoption of fitness tracking and health improvement for office workers. For instance, it has been observed from workplace wellness programs that participants acquired collective fitness tracking behaviors as a result of collaboration on workouts with colleagues [14]. In fact, Human-Computer Interaction (HCI) and Computer-Supported Cooperative Work ( $\mathrm{CSCW}$ ) researchers have investigated the effect of cooperation on fitness-promoting technologies in a variety of contexts. Ahtinen et al. [1] found that connecting with friends or family members with a similar fitness target could strengthen their motivation to engage in exercise. Munson et al. [44] suggested that having shared fitness goals and activity with friends over social media could support positive changes in health behavior. More recently, Chen et al. [11] indicated that having cooperative accountability with a strong tie could lead to improved fitness outcomes for chronically ill patients. Social dynamics for office workers are essential in promoting physical and psychological wellbeing [51]. Previous research has also indicated that sharing fitness data and setting a collective goal among office workers may support fitness tracking in the workplace [14]. Yet, no empirical evidence has actually demonstrated the effect of the cooperative mechanism in fitness promotion among office workers, which leads us to our first research question:

- RQ1: Does cooperative fitness tracking between co-workers improve the level of physical activity?

For cooperative fitness tracking, besides the social effects of peer bonding, we observe that the proximity between the co-workers in the office environment may also affect its outcome. In the CSCW community, the effects of distance have been extensively concerned with workplace technologies and many social practices [4,49]. For instance, an experiment by Bradner and Mark [5] showed that the likelihood of cooperation through communication technologies could be abandoned due to the increase of interpersonal distance. Similarly, Cummings and Kiesler [18] found that greater distance between team members could lead to lower cooperative performance. In our view, however, such classic CSCW narratives of distance effects and the way in which they influence technology-assisted workplace fitness promotion needs to be further explored. It is a worthy topic of study to understand the effects of physical proximity at work on cooperative fitness tracking in the office context. To this end, this study involves two types of co-workers, both those who are distributed and co-located (i.e. at the same site), to explore our second research question:

- RQ2: Does the physical proximity between co-workers influence their adoption of cooperative fitness tracking?

In this study, we propose a peer-based cooperative fitness tracking (PCFT) strategy for fitness promotion in the workplace. We conducted a field study to investigate its effectiveness (RQ1), and the potential impact of interpersonal proximity (RQ2) on this type of collaboration. The study was carried out in three stages: a baseline week, a PCFT intervention week, and a post-intervention week. A fitness tracker named Mi-band [65] was used as a research tool to collect fitness data and implement the PCFT intervention. The aim of the comparison of the fitness data between the three stages was to evaluate the effectiveness of PCFT in promoting physical activity. For this study, we recruited participants who were from same or different 
offices. Based on the physical proximity between the dyads of participants, they were divided into the 'distributed' or the 'co-located' group. The comparison between the two groups examined the effects of the dyad's proximity on the adoption of PCFT. In addition, we collected qualitative data in interviews in order to better understand the user experience with PCFT and identify the challenges involved in blending PCFT into a working routine. This paper makes the following contributions:

- Evidence that PCFT is effective in promoting physical activity for co-located coworkers.

- Design implications that leverage the peer-based cooperative strategy as a motivational factor for fitness promotion in the workplace.

\section{RELATED WORK}

\subsection{Fitness Tracking for Office Workers}

In the $\mathrm{HCI}$ and $\mathrm{CSCW}$ communities, research on fitness tracking technology for office workers has mainly focused on three aspects. The first is embedding sensors into the physical context to enrich fitness tracking in the workplace. For example, HealthSit is a pressure sensor mat that can be placed on an office chair to monitor an office worker's sitting behaviors and provide casual exergames, which facilitate low-back stretch exercising during work breaks [55]. Limber is a DIY kit that contains various wearable sensor packs that are used to monitor the movement of different body parts in order to prevent office injuries [35]. Similarly, Tap-Kick-Click is a pair of smart shoes that tracks foot movements and facilitates foot-based interaction at a standing desk to improve standing postures [58].

The second focus is on integrating fitness tracking technology with the workplace health and wellness programs. This type of research provides deep insights into worker's behavioral changes and subjective opinions with technology-assisted health programs in office settings. For instance, by analyzing data in the literature, Christophersen et al. [13] identified the idea that incentivized activity sharing in the company might actually be problematic for workers' discipline and health goals. From observations of a workplace fitness campaign, Gorm and Shklovski [23] suggested that the privacy concerns of participants in relation to publishing daily steps increased over time. By combining interviews and surveys, Chung et al. [14] observed that it was challenging to employ tracking and fitness behaviors in a busy work schedule. Several studies also suggested that technology-assisted workplace health programs in their current forms may overburden the daily routine and thus create work stress [14,22,23].

The third strand of our research is to investigate motivational factors that are applicable in relation to fitness tracking technology for office workers. This is also the primary objective of this paper. Early studies have explored how to improve self-awareness and encourage spontaneous actions that enhance active lifestyles at both the individual and the social level. For instance, Houston uses the user's walking history data to adjust the fitness goal and thus improves adherence to the health intervention [15]. UbiGarden visualizes user's fitness data with a digital metaphor of flowers to enhance motivation and engagement [17]. Similarly, Breakaway implements ambient feedback to encourage the user to move and reduce sedentary time [28]. Besides increasing self-awareness about being physically active, we also survey the social aspects of fitness tracking and relevant applications designed for office workers.

\subsection{Social Features of Fitness Tracking Technologies}

Most physical activities facilitate social interaction, but even with solo exercises such as walking or jogging, social bonding can motivate individuals to engage with these fitness activities. For 
instance, Stickers for Steps [42] is a fitness promotion application that links daily steps to virtual stickers and increases motivation by encouraging people to collect and exchange the stickers. fogging over a Distance [39] is a co-exercise system that helps create a social experience of jogging together for partners who are geographically apart. The results suggested that such social support could enhance engagement with the exercise and increase the level of activity.

As suggested by [20,48], a variety of social interaction schemes can be employed within HCI that have persuasive design features, including social learning, social recognition, social facilitation, competition, and cooperation. The latter two strategies have been widely used by fitness tracking technologies, supporting users to compete or cooperate with others. It has been concluded that a healthy dose of competition when using fitness tracking technologies is effective in promoting physical activity. For instance, motion-based systems such as Nintendo Wii utilize game mechanisms to facilitate real-time competitions [47]. Nike+ provides sports challenges (e.g., run 100 miles) and leaderboards on the social platform to encourage increased physical activity [46]. Furthermore, research applications such as ShuttleKickers [54] and Kukini [10] have investigated team-based competitions by facilitating groupings of users and teamlevel comparisons. However, excessive competition can produce adverse results $[1,36,38]$. This is why competition mechanics should be applied carefully, e.g., Foster et al. [21] proposed a friendly-competitive intervention to encourage physical activity among office workers.

Recently, a controlled field study by $\mathrm{Chen}$ and $\mathrm{Pu}$ [12] compared the competitive and cooperative mechanisms among people by using the HealthyTogether app. Their results revealed that cooperation was more efficient than competition in promoting physical activity. Thanks to bonding among team members, cooperative settings in social fitness applications can improve health outcomes as well as interpersonal relationships. For instance, Fish'n'Steps [36] uses the growth and emotional state of virtual pets to represent the daily activeness of a group of participants, which reinforces their awareness of being active. Chick Clique [63] generates a collective fitness result by averaging the steps of all team members and allows them to encourage each other by sending text messages. Pass the Ball [56] tracks one member's activity at any one time in relation to the team score, which improves social interaction within the team.

It has been demonstrated that pre-existing social relationships between users can play a critical role in supporting cooperative fitness tracking. Lin et al. [36] identified the fact that anonymous cooperation did not help in improving exercise performance. Instead, according to Munson et al. [44], cooperative physical activities with online friends were able to catalyze support. Moreover, Chen et al. [11] discovered that people with chronic diseases were more motivated to adhere to a shared fitness target with people with whom they have a strong tie than with strangers. For office workers, social bonding between co-workers shows great potential when it comes to enhancing communication and collaboration [30]. In this study, we investigate using a cooperative mechanism for fitness tracking involving two co-workers.

\subsection{The Effects of Proximity for Cooperative Bonding Between Co-Workers}

In office settings, the proximity between co-workers may significantly affect their cooperation and social activities [57]. For instance, the physical presence of colleagues may increase the likelihood of interpersonal support, but too much communication may cause mental overload and is not always welcomed at work [32]. The research about the effects of distance on social activities can be traced back to the 1980s. Latané [33] argued that the degree of social interaction is negatively associated with interpersonal distance. Similarly, Kraut et al. [31] suggested that reducing interpersonal distance could increase communication among people engaged in cooperation. An experiment by Sensenig et al. [59] indicated that, even within one room, close proximity between players could enhance their team performance in a cooperative activity ( the prisoner's dilemma game).

Along with the development of CSCW, the effects of distance have been widely investigated for technology-mediated communication and workplace collaboration. A literature PACM on Human-Computer Interaction, Vol. 2, No. CSCW, Article 146. Publication date: November 2018. 
review by Olson and Olson [49] concluded that physical distance, together with factors such as available technology, affects users' collaborative behaviors. Bradner and Mark's experiment [5] tested the effects of distance on social interaction via video conferencing and instant messaging. Their results suggested that participants' attitude to cooperation is negatively associated with interpersonal distance. Many previous studies also indicated the benefits of close proximity between co-workers in the working context. For instance, Allen [2] suggested that the frequency of communication would be increased when the physical distance between coworkers is less than 30 meters. Hawkey et al. [26] revealed that close proximity between coworkers could contribute to their enjoyment and effectiveness at work if they are engaged in co-located collaboration. Cameron and Webster [9] showed that face-to-face communication at work could offer richer social experience for office workers than using communication technologies. However, few studies have examined the effects of interpersonal proximity in the context of cooperative fitness promotion in the workplace.

Based on the findings from earlier work [12,16], our study design involved using the PCFT strategy to improve the level of physical activity of a dyad of co-workers by enabling them to set a collective fitness goal, track the fitness data, and share progress with each other. We also investigated the effects of their interpersonal distance at work for the adoption of PCFT. Next, we elaborate on our study design in some detail.

\section{THE STUDY}

This section describes the setup of the cooperative fitness tracking application and the research tool employed, as well as the study design used to evaluate the proposed social strategy, including the research hypotheses, the recruitment of participants, and the study procedure.

\subsection{Peer-Based Cooperative Fitness Tracking (PCFT)}

Peer-based cooperative fitness tracking (PCFT) requires two paired co-workers. The core of PCFT is to utilize their social bonding and daily social interaction at work as a tool to promote mutual physical activities. Similar to $[11,12,16]$, PCFT was designed with the following two main features.

- A collective goal: Each dyad could create a cooperative daily step goal that would be fulfilled by the summary of their steps. For example, if a user has made 5000 steps and the buddy has made 6000 steps, in total, they have completed 11000 steps, which is compared to their group goal, e.g., 15000 steps.

- Data sharing: For each dyad, one can check the other's accomplished step data through the fitness tracking application at any time and initiate online interpersonal communication.

We used a fitness tracking device named Xiaomi Mi-band [65] and its app as the research tools to collect the fitness data and implement the PCFT intervention. Specifically, we provided each participant with a Mi-band (see Fig. 1(a)) to collect their daily step data. Xiaomi Mi-band is a smart activity-tracking wristband that is connected to a mobile application named Mi-fit. Here we used Mi-band because of its low cost (approx. $€ 15$ each compared to approx. $€ 90$ for a Fitbit wristband) and ease of maintenance (battery life approx. 30 days compared to approx. 7 days for Fitbit). Throughout the study, participants were asked to wear the Mi-band during waking hours for step tracking. Before the study, participants installed the Mi-fit app on their smartphone. In this study, the Mi-fit app only offers basic social functions supporting two features of PCFT, without introducing additional functions (e.g., hourly/daily notifications, virtual coach, etc.). 

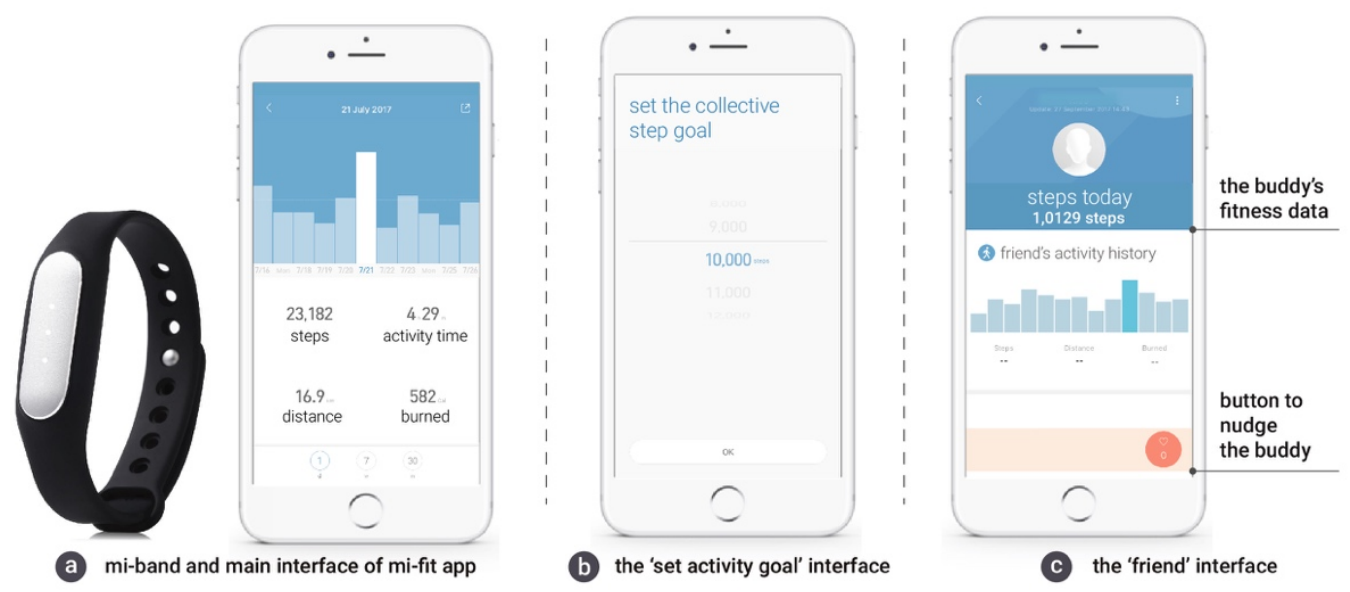

Fig. 1. (a) Overview of the Mi-band and Mi-fit app; (b), (c) Interfaces in the Mi-fit app to facilitate PCFT.

To implement PCFT, the dyad of participants need to set their collective goal in the mi-fit app first using the 'set activity goal' interface in Fig. 1(b). The collective fitness goal was decided based on consensus and could be adjusted at any time during the PCFT intervention. Then, the participants could check their own daily steps via the main interface (see Fig. 1(a)), and also their buddy's daily steps on the 'friend' interface (see Fig. 1(c)). Moreover, within the Mi-fit app, participants could send their buddy a simple system-generated message (a nudge), e.g., "it is time for us to take a walk", as a hint to initiate social interaction related to the collective goal.

\subsection{Research Hypotheses}

We conducted a field study consisting of a baseline week, an intervention week, and a postintervention week. PCFT was applied only in the intervention week. The comparison of daily step data over a period of three weeks was intended to verify whether the proposed PCFT strategy would improve the level of physical activity. Our first hypothesis is:

- H01: Participants' daily steps in the PCFT intervention week will be greater than in the baseline and post-intervention week.

To explore the proximity effects on PCFT, the study recruited dyads with different degrees of proximity at work. The dyads who worked in the same office room were defined as a 'colocated group'. The dyads who worked in the same building but in different office rooms were defined as a 'distributed group'. The comparison of the set goals and the actual step data between the two groups was made to investigate the effects of distance between co-workers in terms of their adoption of the PCFT intervention. The second hypothesis is:

- H02: The co-located participants will adopt PCFT more than the distributed participants.

An additional aim of this study was to understand how PCFT might support physical activity among office workers and identify which factors affect the application of PCFT in the office settings. 


\subsection{Participants}

We recruited participants by spreading information via word of mouth, taking a snowball sampling approach. Initially, we asked people we knew who had similar characteristics to our target subjects. We then asked them to pass the information to their social contacts. During recruiting, we screened study candidates based on the following criteria. First, we selected participants who regularly engaged in office-based work for at least 6 hours per day. Second, the selected participants in each dyad also worked in the same building. Third, we aimed to balance the features of subject between the distributed and the co-located groups. Fourth, we recruited participants who intended to engage in physical activity. This criterion was addressed by using the transtheoretical model (TTM) [53], which identifies an individual's readiness for engaging in health-related behavior according to the following five stages: from pre-contemplation to contemplation, to preparation, to action, to maintenance. By asking them to fill in Physical Activity Questionnaire to Stage of Change [52], we excluded candidates at the pre-contemplation stage because they did not want to become physically more active over the next six months. Lastly, we excluded people who had physical complaints that might make it risky to perform physical activity during our study.

Table 1. Participant characteristics and groupings.

\begin{tabular}{|c|c|c|c|c|c|c|c|}
\hline \multicolumn{4}{|c|}{ (a) 'distributed' group } & \multicolumn{4}{|c|}{ (b) 'co-located' group } \\
\hline dyad & subject & gender & TTM & dyad & subject & gender & TTM \\
\hline \multirow{2}{*}{ P01 } & S01 & female & contemplation & \multirow{2}{*}{ P06 } & S11 & female & contemplation \\
\hline & S02 & male & contemplation & & $\mathrm{S} 12$ & female & preparation \\
\hline \multirow{2}{*}{ P02 } & S03 & male & contemplation & \multirow{2}{*}{ P07 } & S13 & female & maintenance \\
\hline & S04 & male & maintenance & & $\mathrm{S} 14$ & female & maintenance \\
\hline \multirow{2}{*}{ P03 } & S05 & male & maintenance & \multirow{2}{*}{ P08 } & S15 & male & contemplation \\
\hline & S06 & female & maintenance & & $\mathrm{S} 16$ & male & contemplation \\
\hline \multirow{2}{*}{ P04 } & S07 & female & preparation & \multirow{2}{*}{ P09 } & S17 & male & preparation \\
\hline & S08 & female & contemplation & & S18 & female & maintenance \\
\hline \multirow{2}{*}{ P05 } & S09 & male & maintenance & \multirow{2}{*}{ P10 } & S19 & male & maintenance \\
\hline & $\mathrm{S} 10$ & male & maintenance & & $\mathrm{S} 20$ & male & maintenance \\
\hline
\end{tabular}

At the beginning of the experiment, 24 participants were recruited to participate without knowing about the goal of the study. They were also given the opportunity to withdraw at any point. The selected participants were all university employees. Each dyad of participants had been co-workers for at least one year and had very good relationships in their leisure time. Two dyads of participants dropped out due to an unexpected work absence during the study. This paper therefore reports results from the 20 subjects (gender: 11 males and 9 females, age: $M=28.6, S D=2.7, M i n=26, M a x=35)$, who completed the entire field study. Their characteristics are summarized in Table 1 . We labeled the 10 participating dyads as P01, P02 ... P10 and the 20 subjects as $\mathrm{S} 01, \mathrm{~S} 02 \ldots \mathrm{S} 20$.

\subsection{Procedure and Data Collection}

The study was conducted during the Spring in the Netherlands. As shown in Fig. 2, the study was initiated by an introductory session to explain the procedure of the study without discussing the research hypotheses. Afterwards, each participant received a Mi-band wristband as well as instructions to set up the app on their smartphones. During the study, participants wore the wristband to track their physical activity. We collected participants' daily step data over the three-week study period. In the intervention week, the cooperative step goals of the 
participants were recorded on a daily basis. Before the intervention week, we introduced the mechanism of PCFT to participants and enabled the PCFT features (collective goal setting and data sharing) of the Mi-fit app. In the post-intervention week, the PCFT features were disabled so the Mi-fit app worked the same as in the baseline week.

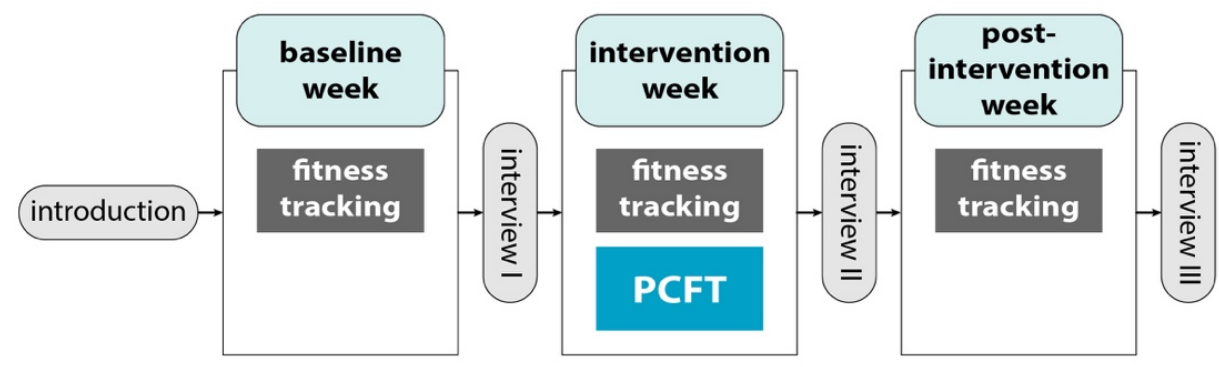

Fig. 2. A visualization of overall study procedure.

At the conclusion of every study week, we conducted an interview with each dyad separately. The interviews followed a pre-set protocol and included open-ended questions about the fitness tracking and its influence on participants' physical activity. First, we asked the participants to interpret their activity data by asking the questions such as "Could you look at your step data and explain what physical activities were related to these data?" We then asked them to elaborate on their experience with fitness tracking in the past week, with questions such as "Could you please share some stories about your experiences related to the fitness-tracking in the past week?" We also asked the participants to explain some interesting statements that emerged during the interview. All interview sessions were audio-taped and transcribed later for qualitative analysis.

\section{FINDINGS}

\subsection{Quantitative Analysis}

Analyses of the quantitative data were conducted using SPSS software, and the results were examined to validate our two research hypotheses. First, distributions were confirmed as not significantly different from the normality (Shapiro-Wilk test $p>0.05$ ). Then a two-way mixed ANOVA was conducted to examine the effects of the PCFT intervention and differing degrees of proximity as well as their interaction. When sphericity could not be assumed, degrees of freedom were adjusted. Where ANOVA was significant, post-hoc analyses were conducted using Bonferroni correction for pairwise comparisons. We analyzed goal commitment to demonstrate the adoption of PCFT. Descriptive analyses were conducted to explore the difference between the co-located and the distributed groups regarding goal setting, compliance, and fulfillment during the intervention week.

\subsubsection{The improvement of fitness behaviors}

We calculated the mean daily steps made by each participant in the baseline, the intervention, and the post-intervention week respectively for the comparative analysis. An increased number of daily steps indicates an improvement in fitness behaviors. Fig. 3(a) shows the changes in the numbers of participants' daily steps across the three study weeks. Compared to the baseline week $(M=7301, S E=586)$, daily steps during the intervention week $(M=7996, S E=565)$ increased by $9.5 \%$ and then returned to $7213(S E=502)$ in the post-intervention week. For the distributed group, the averages of their daily steps were $7184(S E=829)$ in the baseline week, $6743(S E=799)$ in the intervention week, and $6417(S E=710)$ in the post-intervention week. For the co-located 
group, daily steps in the intervention week $(M=9249, S E=799)$ improved greatly compared to the post-intervention week $(M=8010, S E=710)$, and the baseline week $(M=7418, S E=829)$. Fig. 3(b) shows that most participants in the co-located group clearly improved their daily steps during the intervention week. In contrast, the fitness performance of participants from the distributed group varied fitness.

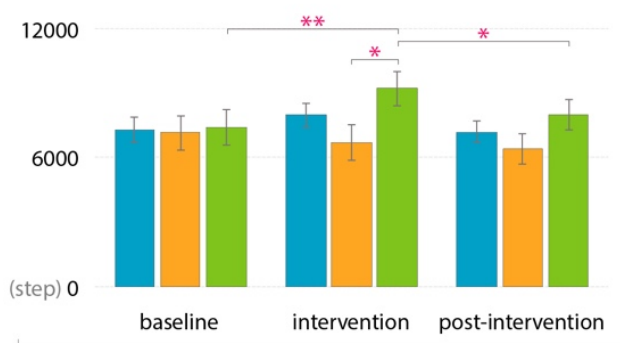

(a) daily averages (step)

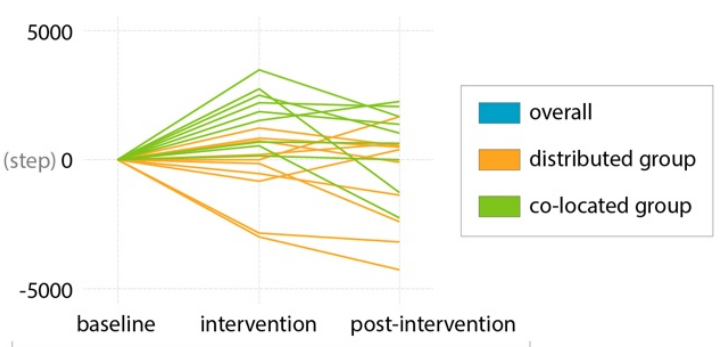

(b) fitness improvements by each subject

Fig. 3. Participants' daily fitness data and improvements across the three study weeks.

To understand the effects of the PCFT intervention and interpersonal proximity in promoting physical activity, we compared participants' step data across the three weeks between the two groups. A two-way mixed ANOVA was conducted with the study week (baseline, intervention, post-intervention) as a within-subjects factor and the proximity of a dyad (co-located vs. distributed) as an independent factor. The analysis revealed that the PCFT intervention had a significant effect on participants' daily steps, $\mathrm{F}(2,36)=3.463, p<0.05$, and that proximity affected the PCFT intervention, $\mathrm{F}(2,36)=6.143, p<0.01$.

Table 2. Pairwise comparisons (a) of distributed group between study stages; (b) of the co-located group between study stages; (c) between two groups in the different study stages. In this table, we define: I = stage 1 baseline; II = stage 2 intervention; III = stage 3 post-intervention.

\begin{tabular}{|c|c|c|c|c|c|c|c|c|c|c|c|}
\hline \multicolumn{4}{|c|}{ (a) distributed } & \multicolumn{4}{|c|}{ (b) co-located } & \multicolumn{4}{|c|}{ (c) co-located vs. distributed } \\
\hline i vs. $\mathbf{j}$ & differ. & $S E$ & $p$ & i vs. $\mathbf{j}$ & differ. & $S E$ & $p$ & phase & differ. & SE & $p$ \\
\hline II vs. I & -440 & 403.93 & 0.871 & II vs. I & 1831 & 403.93 & 0.001 & $\mathrm{I}$ & 235 & 1172.31 & 0.844 \\
\hline III vs. II & -326 & 421.23 & 1.000 & III vs. II & -1240 & 421.23 & 0.026 & II & 2505 & 1129.56 & 0.040 \\
\hline III vs. I & -766 & 545.18 & 0.531 & III vs. I & 591 & 545.18 & 0.877 & III & 1592 & 1003.39 & 0.130 \\
\hline
\end{tabular}

As shown in Table 2, the post-hoc tests with Bonferroni corrections showed that the co-located dyads increased their physical activity significantly with the PCFT intervention compared to the baseline $(p=0.001)$ and the post-study period $(p=0.026)$. No significant differences were found regarding physical activity for the distributed dyads among the three study weeks. In the intervention week with PCFT, the comparison of step data between the two groups revealed that the co-located dyads engaged in significantly more physical activity than the distributed dyads $(p=0.040)$.

\subsubsection{The fitness goal commitment}

During the intervention week, participants set their collective fitness goals, which ranged from 8000 to 18000 steps per dyad on a daily basis, with a mean of $14100(S E=1149.30)$. The average fitness goal for dyads in the distributed group was $13200(S E=1462.87)$ steps. The fitness goals in the co-located group were slightly higher at $15000(S E=1843.91)$ steps per day. This indicated that dyads working in the same office tended to set higher fitness goals with PCFT. 
To examine participants' adherence to their goals during the study, and similar to previous studies [11,37], the frequency of goal compliance and goal fulfillment rate were used as two indices for comparison. We calculated goal compliance by counting how many days a dyad reached their pledged steps for that day, and goal fulfillment rate by the dividing the total steps taken by a dyad (dividend) by their collective goal (divisor) for that day.

Fig. 4 shows that in general our participants achieved their cooperative fitness goal 4.1 days out of $7(S E=0.48, M i n=1, M a x=6)$, and their average goal fulfillment rate was $115 \%, S E=0.05$, Min $=70 \%, M a x=151 \%$. Fig. 4(a) shows that there were four dyads in the co-located group (P06, P07, P08, P09) which achieved their collective goals on more than four days in the intervention week. In contrast, only two of the distributed dyads (P02, P05) achieved this. As shown in Fig. 4 (b), the goal fulfillment rate of four co-located dyads (P06, P07, P08, P09) was above the mean rate of $115 \%$, whereas only one dyad (P5) from the distributed group achieved the fitness goal to a similar extent. These results suggest that the co-located co-workers achieved their fitness goals more frequently and at a higher fulfillment level than the distributed group.

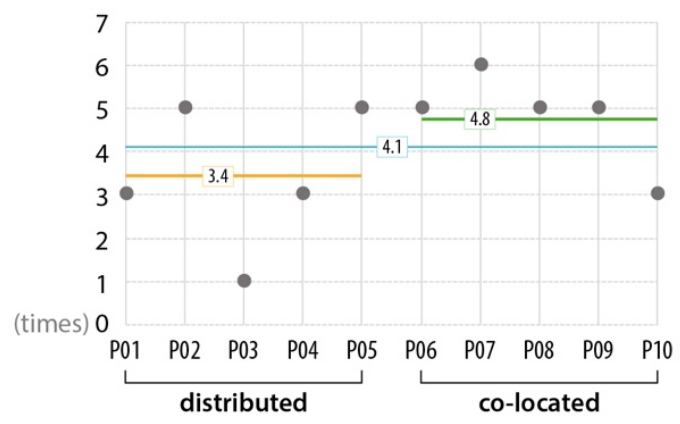

(a) the frequency of goal compliance by each dyad

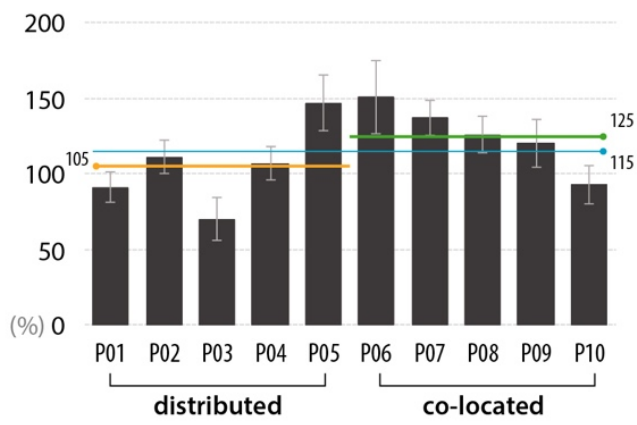

(b) the goal fulfilment rate by each dyad

Fig. 4. Each participating dyad's (a) frequency of achieving the collective goal and (b) Mean and SE of the goal fulfillment rate $(\%)$ in the intervention week.

\subsubsection{Summary}

A $3 \times 2$ ANOVA of participants' daily steps throughout the study period confirmed our first research hypothesis that PCFT can effectively enhance physical activity. This effect was particularly reflected in the significantly increased number of daily steps taken by the co-located group in the intervention week. The comparison between the co-located and distributed groups confirmed our second hypothesis about the positive effect of proximity in supporting the adoption of the PCFT strategy to promote physical activity. Furthermore, the results in relation to the goal commitment indicated that the co-located dyads tended to set higher collective goals, be more motivated to reach these goals, and achieve a higher fulfillment rate than the distributed dyads. To summarize, these quantitative findings suggest that cooperative fitness tracking between two co-workers can promote physical activity, and that close physical proximity at work can facilitate their adherence to the cooperative fitness tracking regime and improving their fitness performance.

\subsection{Qualitative Analysis}

We analyzed the interview data to understand how PCFT supported the co-workers' physical activity and what influenced their adoption of PCFT. We imported all of the interview transcripts into NVivo software and conducted a thematic analysis [6]. To begin with, segmentation of the transcripts was transformed into quote statements and labeled. The labeled 
statements were then measured using inductive coding to identify recurring clusters with emergent themes [62]. After the qualitative analysis, 291 selected quotes were categorized under two main themes: the benefits of PCFT for fitness promotion, and the challenges related to adopting PCFT in the workplace.

\subsubsection{Benefits of PCFT}

The quantitative analyses show that the PCFT intervention can effectively improve the physical activity of the co-workers working in the same office. The interview responses suggest that the PCFT enables a new form of social bonding between two co-workers that extends beyond their existing colleagueship. And this cooperative relationship provides the impetus for their fitness tracking and physical activity. Most participants experienced the PCFT intervention week as positive and reported a frequent knowledge exchange and sharing of experiences with their buddies during work breaks. We elaborate on these findings below, highlighting three aspects.

PCFT facilitated social bonding, which helped to increase physical activity. Although PCFT did not have a decisive impact on the achieving goals, the interview data suggest that PCFT was effective in helping co-workers to establish and maintain a cooperative relationship, which promoted physical activity. Such social incentives improved participants' awareness of fitness tracking and motivated them to contribute to their team fitness goal. Most participants said that they were more likely to check their fitness data during the PCFT week due to curiosity about their buddy's progress. They also reported a strong sense of responsibility for accomplishing the fitness goal, not only for themselves, but also for the collective goal. For instance, one participant (S15) intentionally increased his daily steps to compensate for the unexpected physical injury of his buddy: "From the baseline, we know we took 5000 steps each, and we are eager to get to 16000 steps together. But this week his foot is not that good, so I am the one who is taking more of the load." Although sharing personal data inevitably invited comparisons between the paired partners, none of the participants mentioned that the PCFT had led to an interpersonal competition. Instead, we observed that such comparisons were a motivational factor for improving the level of activity. As S09 said: "His step data was often higher than mine. For me, this is something that can push me to walk more to make a bigger contribution to our goal." By setting fitness goals together and sharing fitness data with each other in a timely fashion, the PCFT tended to make participants more likely to regard their personal improvement as contributing to team performance rather than achieving competitive advantage. For instance, some participants mentioned that "we were working as a team with the same goal" (S08) and "I was more interested in our overall performance" (S10).

PCFT stimulated exchange of knowledge about fitness and health. The cooperative fitness tracking also brought fitness-related topics into daily conversations in the workplace. For instance, S13 stated that "during work breaks, we seemed to talk more about fitness activities than about work." Many participants mentioned they often exchanged exercise experience and fitness knowledge with their PCFT buddy in addition to checking each other's fitness data. For instance, "We begin our talk by asking about each other's progress, then everything would be about how to keep fit" (S15), "I often asked him what he did yesterday to achieve that larger number of steps" (S19). They felt that such frequent discussions improved their awareness of fitness and health. More importantly, some participants believed that such an exchange of knowledge would be beneficial in terms of adjusting their lifestyle in a healthy direction. For instance, S05, a sports enthusiast, told us he often shared fitness tips with his PCFT buddy, e.g., "long hours jogging might hurt your knees, so you'd better train your upper leg muscle to protect them."

PCFT turned a coffee break into a fitness break. In the PCFT intervention week, some participants reported a big change in their work breaks. Instead of having a chat or getting a 
coffee, they tended to take a walk or to do some casual exercises. Coffee breaks seemed to become fitness breaks at work. Specifically, most participants indicated that they were triggered to invite their PCFT buddy to exercise together, e.g., taking a walk during work breaks or a jog after work. As they stated: "in work breaks, we often went out for a walk instead of drinking coffee. Walking together during breaks rarely happened before [PCFT week]" (S07), "I feel more comfortable now asking my colleague 'do you want to go for a walk', as I knew she was up to that" (S14). "In this week [PCFT], we went to the gym together three times after work. It's very natural to ask him to join me" (S16). According to our participants, doing light-intensity physical activity together (mainly walking in this case) during breaks was "more efficient for reaching the goal" (S11) and "beneficial to the social relationship" (S16). In addition, the participants also suggested that when paired partners shared the same work routine or work location, this created more opportunities for them to have a fitness break together. For instance, S19 stated: "On Tuesday I was working with him (S20), and before the lunch-break, he said like oh yeah, we have to reach our goal today so let's have a walk now outside. So, instead of getting a sandwich in the cafeteria, we went to another building to meet our target for steps." On the other hand, it was challenging for the distributed co-workers to adopt PCFT. This is in line with our quantitative findings that the PCFT intervention only significantly increases daily steps when dyads are co-located.

\subsubsection{Challenges to adopt PCFT in the workplace}

In this study, PCFT was developed on the basis of peer-based cooperative mechanisms. The quantitative results showed that the proximity between two co-workers influenced their fitness outcomes with PCFT (co-located vs. distributed group). According to our interview data, we further identified three main challenges for the adoption of PCFT. We suggest that these challenges should be addressed to better leverage PCFT for workplace fitness promotion.

The greater the distance at work, the lower the frequency of fitness breaks together. The participants from the co-located group mentioned that frequent social encounters in the office played a key role in taking a fitness break together at work. As S17 explained: "When we saw each other were less busy, we often asked each other to get a coffee and take a walk by the way, and it happened several times during the day." By contrast, for the participants in the distributed group, they mentioned that it was not always easy to get together for such 'unplanned' social activities, as it was impossible to keep track of each other's availability. This could be one reason why the co-located group achieved greater fitness improvement as well as higher goal compliance and fulfillment rate than the distributed group. Although paired partners can always be reached by the Mi-fit app, greater distance at work still adds to the difficulty of communication between the partners, reducing the frequency of fitness breaks. The presence of the buddy in the same workplace (the same office room) tends to facilitate common social and physical activities. This is why the first challenge when addressing adopting PCFT might be how to reduce the effects of distance and how to how to support the presence of a buddy.

Walking, talking, and inviting, not texting with the app. In the study, we used the Mifit app to support the PCFT intervention. Besides tracking the fitness data, the Mi-fit app also allows the participants to get in touch with their buddy by sending a 'nudging' message. According to our participants, their motivation to use the app for inviting or reminding their buddy to exercise decreased over time. For instance, S09 mentioned: "The first two days we used the app to keep in contact, but it was less afterwards." In contrast, most participants said they would rather "drop by their buddy's office" (S02) and "talk with them in person." (S05). As S10 mentioned: "I prefer to go to his office and ask him to take a walk, although sometimes he was busy with work and couldn't join me, but at least, I was walking instead of texting." As a result, the participants in the distributed group mostly took care of their own fitness with little communication: "The last two days I just did my part without thinking about my colleague's progress" (S04). Co-located participants felt it was more convenient (S20) and efficient (S15) to PACM on Human-Computer Interaction, Vol. 2, No. CSCW, Article 146. Publication date: November 2018. 
discuss the fitness tracking in person than by using the app. These findings suggest that in the co-located group, face-to-face interaction compensated for the limited interaction feature of the app. This also explained why the distributed dyads' intervention adoption and fitness improvement levels were lower than those of the co-located dyads.

The same goal, but a different daily routine. The interview also suggested that a low consistency between the daily routine of paired co-workers might lead to weak bonding for cooperative fitness at work. Some participants described the experience that they tended to ignore the cooperation on fitness tracking when it conflicted with their personal workflow: "If we are working on the same project, it will be easier for us to cooperate on fitness tracking, but sometimes when we work on our own project, the different working time makes it hard to stick to the cooperation"(S08). Additionally, some participants also expressed not only the difference in work routine, but also to the fact that social patterns after work influence cooperative fitness tracking. For instance, some paired participants mentioned that they have a very different life after work, which made it impossible for them to exercise together after work. For instance, S03 mentioned: "We have a totally different lifestyle after work, so it's too hard to exercise together after work", and S13 mentioned "One day she (S14) asked me to go to the gym with her after work, but I needed to go home to cook for my family on that day, so I couldn't join her." They felt that the difference between their daily routines weakened the effects of PCFT to some extent.

\section{DISCUSSION}

Cooperative fitness tracking has been studied in the context of various social relationships, such as strong ties [11], strangers [45], and online social networks [44]. The social bonding between co-workers can be harnessed to develop a cooperative fitness tracking in the workplace [14]. In $\mathrm{CSCW}$, it has been proven that interpersonal distance at work can influence the frequency of communication [2], stressing the effectiveness of collaboration [26], and shared social experience [9]. This paper reports on an empirical study that investigated a peer-based cooperative fitness tracking (PCFT) between co-workers designed to promote workplace fitness and the effects of physical proximity at work on the adoption of PCFT. Comparative analyses of participants' fitness data between the baseline, the intervention, and the post-intervention weeks revealed that the proposed PCFT strategy was effective in improving physical activity. There were better results among the co-located co-workers when it came to fitness-related behavior and goal fulfillment than those distributed across different offices. Our results are consistent with earlier work that indicated the benefits of cooperation for promoting fitness behaviors [12,36,63]. Furthermore, this study provides research evidence that close physical proximity at work could play a significant role in encouraging the uptake of cooperative fitness applications by office workers.

The qualitative results from the interviews indicate that the proposed PCFT may contribute to workplace fitness promotion in three ways. First, PCFT could help to establish and maintain a cooperative relationship between co-workers, which in turn would encourage office workers to become physically more active. Second, PCFT could stimulate the exchange of knowledge related to fitness and health among office workers. Third, PCFT could increase the frequency of work breaks and transform a typical coffee break into a more physically active fitness break. This study also revealed several challenges with the current setup of PCFT. Especially when users are distributed across different workplaces and have very different work routines, it might be hard to form a solid cooperative relationship as the basis for PCFT. This is in line with findings from earlier CSCW studies that distance at work affects cooperative work. According to Olson and Olson [49], the negative effects of distance for cooperation can be mitigated by including effective social interaction design in the collaboration technology. Based on our findings (both from the quantitative and the qualitative analyses), we propose a set of design 
implications to help $\mathrm{HCI}$ and $\mathrm{CSCW}$ researchers, designers and practitioners better leverage PCFT for workplace fitness promotion in future.

\subsection{Design Implications}

\subsubsection{Incorporate social game mechanics into PCFT to enrich fitness breaks}

Our results revealed that people working closely together could meet their collective fitness target more easily due to an increase in the number of work breaks. They tended to incorporate some physical activity into their work breaks. For instance, S19 and S20 often walked to the canteen in another building for lunch; S17 and S18 intentionally walked to a coffee machine farther away so that they could walk a little longer. The current PCFT only supports goal setting, tracking, and sharing of fitness data. Its cooperative mechanism is very simple: it invites the two members of the team to contribute to a pre-set common fitness goal. Although this mechanism may serve as a social incentive for cooperative action, it does not suggest, provide, or assist any cooperative physical activity for fitness breaks. Thus, most of the activity during fitness breaks was limited to walking.

The responses in the interviews suggest that PCFT should introduce more cooperative mechanisms designed to enrich fitness activities during work breaks. For example, some participants suggested embedding game mechanics into PCFT: "It would be nice if this fitness tracking technology could also facilitate some relaxing fitness games with colleagues” (S15). Some game-like challenges and rewards could be embedded into the PCFT-based system to enhance co-located collaborative fitness. One example proposed by Cambo et al. [8] involves encouraging office workers to walk to different locations during work breaks to collect virtual badges. Because office workers prioritized productivity at work, it was felt that collaborative fitness activities or games should add as little burden as possible [14]. Therefore, lightweight collaborative fitness games or activities should be designed and the form of these activities should be well-integrated into the office context.

\subsubsection{Support social presence in the PCFT cooperation relationship}

Studies on social presence theories have shown that rich social cues (e.g., eye contact, posture, dress, and nonverbal cues) during face-to-face communication can greatly enhance intimacy between collaborators [60]. Similarly, in this study, we also observed the positive effects of the face-to-face communication among co-located participants, which actively contributed to PCFT's effectiveness. Based on this finding, we suggest taking the advantages of co-located office workers' proximity into account when introducing PCFT and designing technologies to facilitate social interactions based on their routine overlap. Some forms of notifications could be implemented in PCFT to stimulate co-located social encounters, in which fitness initiatives can be facilitated. For instance, informing users when their buddy is nearby and/or available for a fitness break. Seeing cues on each other's Mi-band might be a simple but direct way to prompt interaction.

For distributed users, we suggest improving awareness of collaboration and enhancing social bonding by making the collective fitness data and goal more visible through ambient displays or peripheral interaction [3]. For example, visualizing the contribution of the cooperative buddy to the fitness goal in the system tray of computers or by the ambient display. Besides fitness tracking and sharing, the PCFT-based application may also need to reduce the effort involved and enrich social cues for remote communication. For instance, developing a PCFT-plugin for office software [41] or a glanceable display for mobile applications [19,24] that presents casual social interactions to enable the paired co-workers to get in touch without disturbing their workflow. Vetere et al. [64] presented several wearable concepts that leverage tactile feedback to support remote interpersonal interactions. Similar features could be developed to support stronger bonding for the distributed PCFT. 


\subsubsection{Fit the PCFT mechanism into the work routine}

According to Jackson [27], routine patterns drive many of our decisions, regardless of the perceived benefits and wish to achieve target behaviors. It has also been shown that daily routine could serve as a means of intervention to shape behavioral change [25]. Previous studies have demonstrated that fitness tracking technologies should fit into personal daily routines [34]. In this paper, PCFT worked by encouraging two co-workers to bond to achieve a collaborative fitness goal. The study revealed that the feasibility of PCFT was not only related to a personal routine, but also to routine consistency between co-workers. The conflict between fitness cooperation and work flow means that PCFT can easily fail. To tackle this issue, 'intelligent' pairing might be applied before setting up cooperative fitness tracking between co-workers, e.g., by analyzing their routine overlap and proximity at work, in order to optimize the feasibility of the intervention [34].

From this study, we found that most participants liked PCFT as an incentive for their collaborative relationships at work, but some of them expressed the view that it was no longer necessary to track their data after work. The boundary between private life and work is an important aspect that must be addressed when designing information technology for office workers. Both earlier work [14,22] and our study involved investigating office workers' fitness tracking practices without separating the work and non-work time. However, our findings suggest that the PCFT and related physical activity should be closely linked to the working routines. For example, peer-based fitness bonding can be selectively enabled or disabled in nonwork time, such as after-work hours and weekends. Moreover, based on [1,11,44], we suggest PCFT can also be applied beyond the workplace context. For instance, outside of work, users could engage in cooperative fitness tracking with their family members, friends, online social networks, etc.

\subsection{Limitations and Future Work}

This was an exploratory study to investigate whether and how the proposed PCFT could encourage physical activity among office workers, as well as understand how physical proximity at work influences the application of PCFT. The findings from our study may need to be cautiously interpreted due to the following limitations. First, the study was conducted in a specific office setting over a relatively short period, which might not be adequate to prove the systemic behavior change in the long-term [29]. Second, as this study focused on a peer-based cooperative strategy as a motivational factor for fitness tracking, the online social interaction implemented in the Mi-fit app was relatively simple. Future work could focus on implementing the design implications reported here into a new PCFT application and investigating its potential in workplace fitness promotion by undertaking longitudinal studies.

\section{CONCLUSIONS}

This paper presents a field study on using cooperative fitness tracking between two co-workers to promote physical activity in the workplace. The proposed PCFT strategy was implemented via a mobile application (Mi-fit) accompanied by an activity tracker (Mi-band) in two ways. First, each dyad of co-workers set a collective goal for daily fitness. Second, the Mi-fit app enabled a social connection so that co-workers could share activity records and interact with each other. The effectiveness of PCFT was examined by comparing the step data between a baseline week, an intervention week, and a post-intervention week. The study also investigated the effects of physical proximity between co-workers at work on the adoption of PCFT. Our quantitative findings show that the PCFT intervention is effective in improving physical activity for the co-located participants, as their step data in the PCFT intervention week were significantly higher than the baseline and post-intervention weeks. The qualitative findings suggest that PCFT could improve participants' awareness of being physically active, stimulate 
exchange of knowledge to support active lifestyles and facilitate fitness breaks in the work routine. Based on these results, we further suggest that future PCFT-based fitness applications could be designed to incorporate social game mechanics as well as support social presence to enhance cooperative bonding and fit better into the work routine of the paired PCFT team.

\section{ACKNOWLEDGMENTS}

The first author of this paper is supported by China Scholarship Council. We are indebted to our participants for their time and effort in this study. Special thanks to Rens Branckaert and Ida Damen for their generous support of this work. We are grateful to the anonymous reviewers whose insightful feedback helped to improve this paper.

\section{REFERENCES}

[1] Aino Ahtinen, Minna Isomursu, Muzayun Mukhtar, Jani Mäntyjärvi, Jonna Häkkilä, and Jan Blom. 2009. Designing social features for mobile and ubiquitous wellness applications. Proceedings of the 8th international Conference on Mobile and Ubiquitous Multimedia, ACM, 12.

[2] Thomas J Allen. 1984. Managing the flow of technology: Technology transfer and the dissemination of technological information within the R\&D organization. MIT Press Books 1 .

[3] Saskia Bakker, Elise van den Hoven, and Berry Eggen. 2015. Peripheral interaction: characteristics and considerations. Personal and Ubiquitous Computing 19, 1: 239-254.

[4] Pernille Bjørn, Morten Esbensen, Rasmus Eskild Jensen, and Stina Matthiesen. 2014. Does distance still matter? Revisiting the CSCW fundamentals on distributed collaboration. ACM Transactions on Computer-Human Interaction (TOCHI) 21, 5: 27.

[5] Erin Bradner and Gloria Mark. 2002. Why distance matters: effects on cooperation, persuasion and deception. Proceedings of the 2002 ACM conference on Computer supported cooperative work, ACM, 226-235.

[6] V. Braun and V. Clarke. 2006. Using Thematic Analysis in Psychology. Qualitative Research in Psychology 3, May 2015: 77-101. http://doi.org/10.1191/1478088706qp063oa

[7] Dena M. Bravata, Crystal Smith-Spangler, Vandana Sundaram, et al. 2007. Using Pedometers to Increase Physical Activity and Improve Health. JAMA 298, 19: 2296. http://doi.org/10.1001/jama.298.19.2296

[8] Scott A. Cambo, Daniel Avrahami, and Matthew L. Lee. 2017. BreakSense: Combining Physiological and Location Sensing to Promote Mobility during Work-Breaks. Proceedings of the $2017 \mathrm{CHI}$ Conference on Human Factors in Computing Systems - CHI '17: 3595-3607. http://doi.org/10.1145/3025453.3026021

[9] Ann Frances Cameron and Jane Webster. 2005. Unintended consequences of emerging communication technologies: Instant messaging in the workplace. Computers in Human behavior 21, 1: 85-103.

[10] Taj Campbell, Brian Ngo, and James Fogarty. 2008. Game design principles in everyday fitness applications. Proceedings of the 2008 ACM conference on Computer supported cooperative work, ACM, 249-252.

[11] Yu Chen, Yunan Chen, Mirana Michelle Randriambelonoro, Antoine Geissbuhler, and Pearl Pu. 2017. Design Considerations for Social Fitness Applications: Comparing Chronically Ill Patients and Healthy Adults. Proceedings of the 2017 ACM Conference on Computer Supported Cooperative Work and Social Computing, ACM, 1753-1762.

[12] Yu Chen and Pearl Pu. 2014. HealthyTogether. Proceedings of the Second International Symposium of Chinese CHI on - Chinese CHI '14, 25-34. http://doi.org/10.1145/2592235.2592240

[13] Mads Christophersen, Peter Mørck, Tue Odd Langhoff, and Pernille Bjørn. 2015. Unforeseen Challenges. International Conference on Universal Access in Human-Computer Interaction, Springer, 288-299.

[14] Chia-Fang Chung, Nanna Jensen, Irina A Shklovski, and Sean Munson. 2017. Finding the right fit: understanding health tracking in workplace wellness programs. Proceedings of the $2017 \mathrm{CHI}$ Conference on Human Factors in Computing Systems, ACM, 4875-4886.

[15] Sunny Consolvo, Katherine Everitt, Ian Smith, and James a. Landay. 2006. Design requirements for technologies that encourage physical activity. Proceedings of the SIGCHI conference on Human Factors in computing systems - CHI '06: 457-466. http://doi.org/10.1145/1124772.1124840

[16] Sunny Consolvo, Predrag Klasnja, David W. McDonald, and James a. Landay. 2009. Goal-setting considerations for persuasive technologies that encourage physical activity. Proceedings of the 4th International Conference on Persuasive Technology - Persuasive '09, 1. http://doi.org/10.1145/1541948.1541960

[17] Sunny Consolvo, David W. McDonald, Tammy Toscos, et al. 2008. Activity Sensing in the Wild: A Field Trial of UbiFit Garden. Proc. of CHI 2008, 1797-1806. http://doi.org/10.1145/1357054.1357335

[18] Jonathon N Cummings and Sara Kiesler. 2005. Collaborative research across disciplinary and organizational boundaries. Social studies of science 35, 5: 703-722.

[19] Andrey Esakia, D Scott McCrickard, Samantha Harden, and Michael Horning. 2018. FitAware: Promoting Group Fitness Awareness ThroughGlanceable Smartwatches. Proceedings of the 2018 ACM Conference on Supporting Groupwork, ACM, 178-183. 
[20] BJ Fogg. 2003. Persuasive technology: using computers to change what we think and do. Morgan Kaufmann Publishers. http://doi.org/10.4017/gt.2006.05.01.009.00

[21] Derek Foster, Conor Linehan, Ben Kirman, Shaun Lawson, and Gary James. 2010. Motivating physical activity at work: using persuasive social media for competitive step counting. MindTrek 2010: 111-116. http://doi.org/10.1145/1930488.1930510

[22] Nanna Gorm and Irina Shklovski. 2016. Steps, Choices and Moral Accounting: Observations from a Step-Counting Campaign in the Workplace. Proceedings of the 19th ACM Conference on Computer-Supported Cooperative Work \&amp; Social Computing: 148-159. http://doi.org/10.1145/2818048.2819944

[23] Nanna Gorm and Irina Shklovski. 2016. Sharing steps in the workplace: Changing privacy concerns over time. Proceedings of the 2016 CHI Conference on Human Factors in Computing Systems, ACM, 4315-4319.

[24] Rúben Gouveia, Fábio Pereira, Evangelos Karapanos, Sean A Munson, and Marc Hassenzahl. 2016. Exploring the design space of glanceable feedback for physical activity trackers. Proceedings of the 2016 ACM International Joint Conference on Pervasive and Ubiquitous Computing, ACM, 144-155.

[25] Kirsten Gram-Hanssen. 2008. Consuming technologies - developing routines. Journal of Cleaner Production 16, 11: 1181-1189. http://doi.org/10.1016/j.jclepro.2007.08.006

[26] Kirstie Hawkey, Melanie Kellar, Derek Reilly, Tara Whalen, and Kori M Inkpen. 2005. The proximity factor: impact of distance on co-located collaboration. Proceedings of the 2005 international ACM SIGGROUP conference on Supporting group work, ACM, 31-40.

[27] Tim Jackson. 2005. Motivating Sustainable Consumption - A review of evidence on consumer behaviour and behavioural change. http://doi.org/10.1260/0958305043026573

[28] Nassim Jafarinaimi, Jordi Forlizzi, Amy Hurst, and John Zimmerman. 2005. Breakaway: An Ambient Display Designed to Change Human Behavior. CHI'05 extended abstracts on Human factors in computing systems: 19451948. http://doi.org/10.1145/1056808.1057063

[29] Predrag Klasnja, Sunny Consolvo, and Wanda Pratt. 2011. How to evaluate technologies for health behavior change in HCI research. Proceedings of the SIGCHI Conference on Human Factors in Computing Systems, 30633072. http://doi.org/10.1145/1978942.1979396

[30] Kathy E Kram and Lynn A Isabella. 1985. Mentoring alternatives: The role of peer relationships in career development. Academy of management Journal 28, 1: 110-132.

[31] Robert E Kraut, Robert S Fish, Robert W Root, and Barbara L Chalfonte. 1990. Informal communication in organizations: Form, function, and technology. Human reactions to technology: Claremont symposium on applied social psychology, Citeseer, 145-199.

[32] Robert E Kraut, Susan R Fussell, Susan E Brennan, and Jane Siegel. 2002. Understanding effects of proximity on collaboration: Implications for technologies to support remote collaborative work. Distributed work: 137-162.

[33] Bibb Latané. 1981. The psychology of social impact. American psychologist 36, 4: 343.

[34] Amanda Lazar, Christian Koehler, Joshua Tanenbaum, and David H. Nguyen. 2015. Why we use and abandon smart devices. Proceedings of the 2015 ACM International Joint Conference on Pervasive and Ubiquitous Computing - UbiComp '15: 635-646. http://doi.org/10.1145/2750858.2804288

[35] Ken Leung, Derek Reilly, Kate Hartman, Suzanne Stein, and Emma Westecott. 2012. Limber: DIY wearables for reducing risk of office injury. Proceedings of the Sixth International Conference on Tangible, Embedded and Embodied Interaction, ACM, 85-86.

[36] James J Lin, Lena Mamykina, Silvia Lindtner, Gregory Delajoux, and Henry B Strub. 2006. Fish'n'Steps: Encouraging Physical Activity with an Interactive Computer Game. In UbiComp 2006: Ubiquitous Computing. 261-278. http://doi.org/10.1007/11853565_16

[37] Edith Talina Luhanga, Akpa Akpro Elder Hippocrate, Hirohiko Suwa, Yutaka Arakawa, and Keiichi Yasumoto. 2018. Identifying and Evaluating User Requirements for Smartphone Group Fitness Applications. IEEE Access 6: 3256-3269.

[38] Andrew Macvean and Judy Robertson. 2013. Understanding exergame users' physical activity, motivation and behavior over time. Proceedings of the SIGCHI Conference on Human Factors in Computing Systems - CHI '13, 1251. http://doi.org/10.1145/2470654.2466163

[39] James A. Martin. 2014. Pros and Cons of Using Fitness Trackers for Employee Wellness. Cio. Retrieved from http://www.cio.com/article/2377723/it-strategy/pros-and-cons-of-using-fitness-trackers-for-employeewellness.html?page $=2$

[40] Ray M Merrill, Beverly Hyatt, Steven G Aldana, and Dan Kinnersley. 2011. Lowering employee health care costs through the healthy lifestyle incentive program. Journal of Public Health Management and Practice 17, 3: 225232.

[41] Dan Morris, a.J. Bernheim Brush, and Brian R. Meyers. 2008. SuperBreak: using interactivity to enhance ergonomic typing breaks. Proceeding of the twenty-sixth annual $\mathrm{CHI}$ conference on Human factors in computing systems - CHI '08, 1817. http://doi.org/10.1145/1357054.1357337

[42] Alistair Morrison and Viktor Bakayov. 2017. Stickers for steps: a study of an activity tracking system with face-toface social engagement. Proceedings of the ACM on Human-Computer Interaction 1, CSCW.

[43] Florian Mueller, Frank Vetere, Martin R Gibbs, Darren Edge, Stefan Agamanolis, and Jennifer G Sheridan. 2010. Jogging over a distance between Europe and Australia. Proceedings of the 23nd annual ACM symposium on User interface software and technology, ACM, 189-198. 
[44] Sean Munson, Erin Krupka, Caroline Richardson, and Paul Resnick. 2015. Effects of public commitments and accountability in a technology-supported physical activity intervention. Proceedings of the 33rd Annual ACM Conference on Human Factors in Computing Systems, ACM, 1135-1144.

[45] Sean Munson and Sunny Consolvo. 2012. Exploring Goal-setting, Rewards, Self-monitoring, and Sharing to Motivate Physical Activity. Proceedings of the 6th International Conference on Pervasive Computing Technologies for Healthcare: 25-32. http://doi.org/10.4108/icst.pervasivehealth.2012.248691

[46] Nike. Nike+Training App. https://goo.gl/9kWN4y.

[47] Nintendo. Nintendo Wii. http://wii.com.

[48] Harri Oinas-Kukkonen and Marja Harjumaa. 2009. Persuasive systems design: Key issues, process model, and system features. Communications of the Association for Information Systems 24, 1: 485-500.

[49] Gary M Olson and Judith S Olson. 2000. Distance matters. Human-computer interaction 15, 2: 139-178.

[50] Neville Owen, Geneviève N Healy, Charles E Matthews, and David W Dunstan. 2010. Too much sitting: the population health science of sedentary behavior. Exercise and sport sciences reviews 38, 3: 105-113. http://doi.org/10.1097/JES.0b013e3181e373a2

[51] Neville Owen, Takemi Sugiyama, Elizabeth E Eakin, Paul A Gardiner, Mark S Tremblay, and James F Sallis. 2011. Adults' sedentary behavior: determinants and interventions. American journal of preventive medicine 41, 2: 189196.

[52] J O Prochaska. 1991. exercise: stages of change (short form). Retrieved from http://web.uri.edu/cprc/exercisestages-of-change-short-form/

[53] J O Prochaska and W F Velicer. 1997. The transtheoretical model of health behavior change. American journal of health promotion: AJHP 12, 38-48. http://doi.org/10.4278/0890-1171-12.1.38

[54] Xipei Ren, Yuan Lu, Aarnout Brombacher, and Tilde Bekker. 2016. ShuttleKickers: Exploring Social Persuasions to Encourage Physical Activities. Proccedings of the 30th BCS-HCI British Human Computer Interaction Conference, 1-11. http://doi.org/10.14236/ewic/HCI2016.19

[55] Xipei Ren, Bin Yu, Yuan Lu, Yu Chen, and Pearl Pu. 2018. HealthSit: Designing Posture-Based Interaction to Promote Exercise during Fitness Breaks. International Journal of Human-Computer Interaction. http://doi.org/10.1080/10447318.2018.1506641

[56] John Rooksby, Mattias Rost, Alistair Morrison, and Matthew Chalmers. 2015. Pass the ball: enforced turn-taking in activity tracking. Proceedings of the 33rd Annual ACM Conference on Human Factors in Computing Systems, ACM, 2417-2426.

[57] Kerstin Sailer and Ian McCulloh. 2012. Social networks and spatial configuration-How office layouts drive social interaction. Social networks 34, 1: 47-58.

[58] William Saunders and Daniel Vogel. 2016. Tap-Kick-Click: Foot Interaction for a Standing Desk. Proceedings of the 2016 ACM Conference on Designing Interactive Systems, 323-333. http://doi.org/10.1145/2901790.2901815

[59] John Sensenig, Thomas E Reed, and Jerome S Miller. 1972. Cooperation in the prisoner's dilemma as a function of interpersonal distance. Psychonomic Science 26, 2: 105-106.

[60] John Short, Ederyn Williams, and Bruce Christie. 1976. The social psychology of telecommunications.

[61] Olivia Solon. 2015. Wearable Technology Creeps Into The Workplace. Bloomberg.com: 1-6. Retrieved from http://www.bloomberg.com/news/articles/2015-08-07/wearable-technology-creeps-into-the-workplace

[62] David R Thomas. 2006. A general inductive approach for analyzing qualitative evaluation data. American journal of evaluation 27, 2: 237-246.

[63] Tammy Toscos, Anne Faber, Shunying An, and Mona Praful Gandhi. 2006. Chick clique: persuasive technology to motivate teenage girls to exercise. CHI '06 Extended Abstracts on Human Factors in Computing Systems, 18731878. http://doi.org/10.1145/1125451.1125805

[64] Frank Vetere, Martin R Gibbs, Jesper Kjeldskov, et al. 2005. Mediating intimacy: designing technologies to support strong-tie relationships. Proceedings of the SIGCHI conference on Human factors in computing systems, ACM, 471-480.

[65] Xiaomi. 2016. Mi Band. Website. Retrieved from http://www.mi.com/en/miband/ 